\title{
Temperaturedependence of minimum resource requirements alters competitive hierarchies in phytoplankton
}

LewingtonPearce, Leah; Narwani, Anita; Thomas, Mridul; Kremer, Colin T.; Vogler, Helena; Kratina, Pavel

\section{Published in:}

Oikos

Link to article, DOI:

10.1111/oik.06060

Publication date:

2019

Document Version

Peer reviewed version

Link back to DTU Orbit

Citation (APA):

LewingtonPearce, L., Narwani, A., Thomas, M., Kremer, C. T., Vogler, H., \& Kratina, P. (2019). Temperature dependence of minimum resource requirements alters competitive hierarchies in phytoplankton. Oikos, 128, 1194-1205. https://doi.org/10.1111/oik.06060

\section{General rights}

Copyright and moral rights for the publications made accessible in the public portal are retained by the authors and/or other copyright owners and it is a condition of accessing publications that users recognise and abide by the legal requirements associated with these rights.

- Users may download and print one copy of any publication from the public portal for the purpose of private study or research.

- You may not further distribute the material or use it for any profit-making activity or commercial gain

- You may freely distribute the URL identifying the publication in the public portal

If you believe that this document breaches copyright please contact us providing details, and we will remove access to the work immediately and investigate your claim 
Temperature-dependence of minimum resource requirements alters competitive hierarchies in phytoplankton

Leah Lewington-Pearce ${ }^{1}$, Anita Narwani ${ }^{2}$, Mridul K. Thomas ${ }^{3}$, Colin T. Kremer ${ }^{4,5}$, Helena Vogler ${ }^{2}$ and Pavel Kratina ${ }^{1}$

${ }^{1}$ School of Biological and Chemical Sciences, Queen Mary Univ. of London, London, E1 4NS, UK

${ }^{2}$ Dept of Aquatic Ecology, Swiss Federal Inst. of Aquatic Science and Technology, CH-8600 Dübendorf, Switzerland

${ }^{3}$ Centre for Ocean Life, DTU Aqua, Technical Univ. of Denmark, Lyngby, Denmark

${ }^{4}$ Dept of Ecology and Evolutionary Biology, Yale Univ., New Haven, CT, USA

${ }^{5}$ W. K. Kellogg Biological Station, Michigan State Univ., Hickory Corners, MI, USA

Corresponding author: Pavel Kratina, School of Biological and Chemical Sciences, Queen Mary Univ. of London, London, E1 4NS, UK. E-mail: p.kratina@qmul.ac.uk

Decision date: 05-Mar-2019

This article has been accepted for publication and undergone full peer review but has not been through the copyediting, typesetting, pagination and proofreading process, which may lead to differences between this version and the Version of Record. Please cite this article as doi: [10.1111/oik.06060].

'This article is protected by copyright. All rights reserved.' 


\section{ABSTRACT}

Resource competition theory is a conceptual framework that provides mechanistic insights into competition and community assembly of species with different resource requirements. However, there has been little exploration of how resource requirements depend on other environmental factors, including temperature. Changes in resource requirements as influenced by environmental temperature would imply that climate warming can alter the outcomes of competition and community assembly.

We experimentally demonstrate that environmental temperature alters the minimum light and nitrogen requirements - as well as other growth parameters - of six widespread phytoplankton species from distinct taxonomic groups. We found that species require the most nitrogen at the highest temperatures while light requirements tend to be lowest at intermediate temperatures, although there are substantial interspecific differences in the exact shape of this relationship.

We also experimentally parameterize two competition models, which we use to illustrate how temperature, through its effects on species' traits, alters competitive hierarchies in multispecies assemblages, determining community dynamics.

Developing a mechanistic understanding of how temperature influences the ability to compete for limiting resources is a critical step towards improving forecasts of community dynamics under climate warming.

Keywords: climate warming, minimum resource requirements, resource competition theory, chemostat model. 


\section{INTRODUCTION}

Resource availability and environmental temperature exert strong control on biological processes across all scales, from individual metabolism and population growth to community structure and ecosystem functioning (Eppley 1972, Tilman 1982, Sterner and Elser 2002, Brown et al. 2004). Species’ resource-dependent growth rates can be used to develop and apply resource competition theory (Tilman 1982, Chase and Leibold 2003), whereas the temperature-dependence of species' metabolic rates can explain population, community and bulk ecosystem metabolism (Brown et al. 2004, Yvon-Durocher et al. 2010). While the independent influences of resources and temperature on ecological systems are relatively well understood, each alone leaves substantial variation in community dynamics unexplained. This highlights the fact that little is known about how these drivers combine to shape community assembly, despite some indication of their interactive effects on population growth rates (Thomas et al. 2017), competitive dominance (Tilman 1981), and community composition (Hillebrand 2011, Kratina et al. 2012).

The resource-dependence of population growth rate drives species' competitive abilities, one of the principal forces underlying community structure and dynamics (Keddy 2002). Competition for resources has been modelled in numerous phenomenological ways, for example by using interaction coefficients (Chesson 2000) or the degree of resource-use overlap (Macarthur and Levins 1967), but adopting resource competition theory $(\mathrm{RCT})$ has an advantage of explicitly modelling competition as a function of species' resource-dependent growth rates (Tilman 1982, Chase and Leibold 2003). One of the key outcomes of RCT is that the species that survives at the lowest level of the limiting resource outcompetes other species in an environment with constant resource supply (Tilman 1977, 1980, 1982, Miller et al. 2005). This minimum level of resource required to maintain a break-even population growth rate is therefore an important parameter, known as $R^{*} . R^{*}$ and related parameters of resource competition models have been used to predict the outcomes of competition under constant environment in the laboratory, and more recently, also in natural ecosystems (Miller et al. 2005, Dybzinski and Tilman 2007, Edwards et al. 2013).

Environmental temperature places fundamental constraints on organismal metabolism, with effects scaling from individual physiology to the ecology of entire communities (Eppley 1972, Brown et al. 2004, Kingsolver 2009, Dell et al. 2011, Kratina et al. 2012, Sentis et al. 2017). The metabolic theory of ecology posits that the temperature-dependence of an organism's metabolic rate is determined by the 
most rate-limiting underlying biochemical reaction (Gillooly et al. 2001). Scaling up, the temperaturedependence of a population or community's metabolic rate is the aggregate of the contributions of individuals or species, respectively (Savage et al. 2004, Cross et al. 2015). Previous works have used different temperature-dependencies of photosynthesis and respiration (Allen et al. 2005, Schaum et al. 2017) to predict the strength of consumer-resource interactions (O'Connor 2009, Yvon-Durocher et al. 2010), community structure and ecosystem function (Kratina et al. 2012, Shurin et al. 2012) under future climate warming. Temperature can also have differential impacts on various resource uptake and assimilation pathways (Toseland et al. 2013, Daines et al. 2014), which can have knock-on effects on competitive interactions. For example, reaction rates of phosphorus-rich ribosomes are more temperaturesensitive than nitrogen-rich photosynthetic proteins, suggesting that climate warming may shift elemental stoichiometry and resource requirements (Martiny et al. 2013, Yvon-Durocher et al. 2015, Yuan and Chen 2015). Although previous study has experimentally tested the effect of temperature on competition between the pairs of species (Bestion et al. 2018), how temperature influences species minimum resource requirements and competitive hierarchies in multispecies assemblages remains poorly understood.

The temperature-dependence of $\mathrm{R} *$ (the minimum resource level needed to maintain a population) has been postulated for nearly four decades. For any given species, it is assumed that $R^{*}$ is minimized at an intermediate temperature of the species' temperature niche, and increases steeply on either side, following an approximately U-shaped response curve (Lehman and Tilman 2000, Tilman 2004, Thomas et al. 2017). However, very few empirical examples have actually quantified the relationship between $\mathrm{R}^{*}$ and temperature. These experimental examples are limited to the silica and phosphorus requirements of individual diatom species (Tilman 1981, van Donk and Kilham 1990, Shatwell et al. 2014) and to two rotifer species feeding on algae (Stelzer 1998). This lack of empirical data across a range of resources and taxonomic groups critically constrains our mechanistic understanding of community assembly and our ability to understand how warming structures ecological communities.

Here, we investigated the temperature-dependence of phytoplankton resource requirements, because phytoplankton are globally important primary producers, accounting for nearly half of all primary production and supporting consumers across many ecosystems (Field et al. 1998). Phytoplankton rely on a limited number of essential resources for survival and reproduction, including light and macronutrients. Furthermore, phytoplankton competitive and thermal traits have been extensively studied, and are amenable to measurements of resource requirements and temperature-dependent population 
growth rates (Wilson et al. 2007, Kremer et al. 2017a, Thomas et al. 2016, Thomas et al. 2017). Thus, we experimentally tested how temperature influences the traits that govern species' competition for resources, with special emphasis on $R^{*}$. We quantified the temperature-dependence of competitive traits for six common and widely distributed phytoplankton taxa. We then parameterized competition models with these trait estimates to show that the observed temperature-dependences of the key parameters may alter the outcomes of competition between these taxa for limiting resources (light and nitrogen). We aimed to address the following questions: i) How do $R^{*}$ and other traits relating resources to growth rates vary across a temperature gradient? ii) Does interspecific variation in the temperature-dependence of $R^{*}$ and other growth parameters imply that temperature change will influence competitive hierarchies and temporal dynamics of phytoplankton communities?

\section{METHODS}

\subsection{Quantifying resource- and temperature-dependent growth rates}

To investigate the temperature-dependence of resource competition for light and nitrogen, we measured population growth rates of six species spanning three groups of freshwater phytoplankton: cyanobacteria, chlorophytes and diatoms (Table A1). We refer to species by their genus name for simplicity. We estimated their growth rates in two separate experiments that crossed gradients of temperature with: (i) light, and (ii) nitrogen. Prior to each experiment, species were maintained in batch culture in a modified sterile COMBO freshwater medium which did not contain animal trace elements or vitamins (Kilham et al. 1998).

We estimated the growth rates of each species at each of ten levels of nitrogen and light by measuring changes in chlorophyll- $a$ fluorescence over time. We also estimated phycocyanin fluorescence for the cyanobacteria species during the nitrogen experiment, as phycocyanin is a more sensitive measure of cyanobacteria growth (unpublished data). We took daily measurements of these proxies for phytoplankton biomass using a Biotek Cytation 5 multi-mode plate reader. We measured chlorophyll- $a$ fluorescence at excitation and emission wavelengths of $435 \mathrm{~nm}$ and $685 \mathrm{~nm}$. We measured the phycocyanin using excitation and emission wavelength of $620 \mathrm{~nm}$ and $665 \mathrm{~nm}$. Experimental units were tissue-culture plates that were sealed with Breathe-Easy ${ }^{\mathrm{TM}}$ membranes to prevent evaporative losses and cross-contamination between adjacent wells. To reduce the risk of contamination, all acclimation and experimental inoculation steps were performed in a laminar flow hood using sterile technique. Well-

'This article is protected by copyright. All rights reserved.' 
plates were randomly assigned a location within a grid in the temperature-controlled incubators (Multitron, Infors HT, Switzerland), which were set to rotate at $100 \mathrm{rpm}$. Cultures were illuminated at $140.6 \mu \mathrm{mol}$ photons $\mathrm{m}^{-2} \cdot \mathrm{s}^{-1}$ of photosynthetically active radiation (PAR), except for the light-limited treatments (see below), for a 18L:6D photoperiod and maintained at $15^{\circ} \mathrm{C}, 20^{\circ} \mathrm{C}, 25^{\circ} \mathrm{C}$ or $30^{\circ} \mathrm{C}$. These temperatures encompassed the approximate range of each species' previously-estimated optimal temperature for growth $\left(T_{o p t}\right)$ (Thomas et al. 2016).

\subsubsection{Experiment 1: Temperature-dependence of light limitation}

In the light limitation experiment, we factorially manipulated temperature (four levels) and light (ten levels). Sub-cultures of each phytoplankton species were acclimated to the four experimental temperatures and the ten light levels $(0.15,0.95,3.6,6.8,18.7,29.3,49.2,77.3,105.5,140.6 \mu \mathrm{mol}$ photons $\mathrm{m}^{-2} \cdot \mathrm{s}^{-1}$ ) for six days prior to the start of the experiment. Before inoculating each species into the final growth rate experiment, population biomass was estimated with chlorophyll- $a$ fluorescence as a proxy, in order to equalize the starting values across all treatment combinations using dilutions. We measured raw fluorescence units (RFU) of chlorophyll- $a$ by pipetting $1 \mathrm{~mL}$ samples of each acclimated culture into 48-well tissue-culture plates. Dilutions were conducted to achieve a starting $\mathrm{RFU} \leq 1,500$.

The light requirements were estimated by inoculating $100 \mu \mathrm{L}$ of diluted, acclimated phytoplankton culture into $900 \mu \mathrm{L}$ of sterile COMBO medium in a 48 -well Falcon tissue-culture plate to achieve an initial biomass of $\leq 150 \mathrm{RFU}$. This meant that growth rates were estimated from the population biomasses far below the carrying capacity. We used neutral density filters (Solar Graphics ${ }^{\mathrm{TM}}$, Clearwater, Florida) to manipulate the total amount of light supplied without changing light spectrum.

The light filters on the opaque frames prevented unmeasured light from entering the wells from the sides of the plates. Experimental light intensities under the filters were measured using a Skye PAR Quantum sensor.

Measurements of population-level RFU were made in two replicate wells for all temperature and light combinations daily for 10 days. Temperature treatments were applied in two temporal blocks. The $20{ }^{\circ} \mathrm{C}$ treatment was repeated in both blocks as a control for the effect of block, i.e. the $20^{\circ} \mathrm{C}$ treatment was replicated four times (twice in each block). The growth rate estimates at controlled $20^{\circ} \mathrm{C}$ did not differ between blocks. In total we estimated 600 growth rates from 6,000 biomass measurements.

'This article is protected by copyright. All rights reserved.' 


\subsubsection{Experiment 2: Temperature-dependence of nitrogen limitation}

In the nitrogen limitation experiment, we factorially manipulated temperature (four levels) and the concentration of elemental nitrogen in the form of nitrate, $\mathrm{NaNO}_{3}(1,4,6,10,40,60,100,400,600$, $\left.1000 \mu \mathrm{mol} \mathrm{N} \cdot \mathrm{L}^{-1}\right)$. These nitrate concentrations were derived from the experimental estimates of resource limitation of freshwater phytoplankton (Narwani et al. 2015) and additional pilot experiments where we estimated minimum resource requirements for the six focal species. For comparison, standard COMBO media (Kilham et al. 1998) contains $1,000 \mu \mathrm{mol} \cdot \mathrm{L}^{-1}$ of $\mathrm{NaNO}_{3}$.

Sub-cultures of each phytoplankton species were acclimated to all temperature and nitrate combinations for 13 days prior to the start of the experiment (see Supplementary material A2). We first diluted the acclimated cultures to $500 \mathrm{RFU}$ or less, and then inoculated $1 \mathrm{~mL}$ of the cultures with $9 \mathrm{~mL}$ of sterile COMBO containing the assigned nitrogen level into 6-well tissue culture plates, achieving an initial biomass of less than $50 \mathrm{RFU}$. This meant that growth rates were estimated from the population biomasses far below the carrying capacity. We measured population biomass of all species in three replicated wells and calculated their means at all temperature and nitrogen combinations daily over 9 days. This resulted in 720 growth rate estimates from 6,480 biomass measurements.

\subsubsection{Models of population growth}

We described variation in light-dependent growth using a modified version of the Eilers-Peeters model (Eilers and Peeters 1988):

$$
\mu(I)=\frac{\mu_{\max } I}{\frac{\mu_{\max }}{\alpha I_{o p t}^{2}} I^{2}+\left(1-2 \frac{\mu_{\max }}{\alpha I_{\text {opt }}}\right) I+\frac{\mu_{\max }}{\alpha}}+h,
$$

where $\mu$ is the specific growth rate (per day) as a function of irradiance $I$ (in $\mu$ mol photons $\mathrm{m}^{-2} \mathrm{~s}^{-1}$ ), $I_{\mathrm{opt}}$ is the optimal irradiance for growth, and $\alpha$ is the initial slope of the curve. We modified the Eilers-Peeters model by adding the new parameter $h$ (for heterotrophy) to avoid the incorrect assumption that species growth rate is precisely zero in the absence of light $(I=0)$. In purely autotrophic species, the lack of cell growth in the absence of light, in combination with background mortality, leads to a negative specific growth rate. This $h$ parameter is negative in these autotrophic species, reflecting negative growth rate at zero light. In contrast, mixotrophic species may show negative or positive specific growth rates in the absence of light, as a result of the balance between heterotrophic growth and background mortality. For these mixotrophic species, $h$ may be negative or positive (or zero, in which case the equation is identical 
to the Eilers-Peeters model). Note that when $h=0, \mu_{\max }$ is the maximum specific growth rate; otherwise the estimated maximum growth rate is the sum of $\mu_{\max }$ and $h$.

We described variation in nitrogen-dependent growth using a modified version of the Monod equation (Monod 1949):

$$
\mu(N)=\frac{\mu_{\max } N}{N+\frac{\mu_{\max }}{\alpha}}-m
$$

where $\mu$ is the specific growth rate (per day) as a function of nitrogen concentration $N$ (in $\mu \mathrm{mol} \cdot \mathrm{L}^{-1}$ ), $\alpha$ is the initial slope of the curve, $m$ is the background mortality rate (i.e. the specific growth rate at $N=0$ ), and $\mu_{\max }$ is the maximum growth rate only when $m=0$. As with the Eilers-Peeters model, we modified the Monod equation to avoid the assumption that growth rate is zero in the absence of resources $(N=0)$. We did this by subtracting the $m$ parameter (for mortality), because background mortality should lead to specific growth rates that are negative in the absence of nutrients. The estimated maximum growth rate $\left(\mu_{\max }\right)$ is therefore the sum of $\mu_{\max }$ and $m$. Note that the distinction between the $h$ and $m$ parameters in equations (1) and (2) is that the possibility of heterotrophic growth allows for positive or negative growth in the absence of light (captured by the parameter $h$ ), but growth in the absence of nutrients (captured by the parameter $m$ ) is always negative.

We fit equations (1) and (2) to our experimental data (see supplementary material A2), and used the fitted growth curves to estimate $R^{*}$ values. $R^{*}$ is the resource (irradiance or nitrate) level at which each species' specific growth rate is zero. We estimated $R^{*}$ (i.e. $I^{*}$ and $N^{*}$ for light and nutrients respectively) from each fitted growth curve by first numerically estimating the value at which the growth rate was zero. In cases where specific growth rate is negative or zero at a resource level of zero, this estimated value is identical to the $R^{*}$. In cases where specific growth rate is positive at a resource level of zero (i.e. there is detectable heterotrophic growth), the estimated value is no longer the $R^{*}$. This is because the $R^{*}$ is bounded at zero by definition, being a measure of resource availability. Therefore, where these numerical estimates were negative, we set $R^{*}$ to be zero.

\subsection{Temperature-dependence of competition traits}

We characterized the minimum light $\left(I^{*}\right)$ and nitrogen $\left(N^{*}\right)$ requirements, the maximum specific growth rates $\left(\mu_{\max }\right)$, the initial slope of the growth-light curve $(\alpha)$, the specific growth rates at $I=0$, implying heterotrophic growth $(h)$, and the optimal irradiance for growth $\left(I_{\mathrm{opt}}\right)$. We applied two 
approaches to characterize the shape of the temperature-dependence of competitive traits around their maxima (or minima for $R^{*}$ ). First, to characterize the shape of the temperature response curve, we fit a generalized additive mixed model (GAMM). In contrast to GAM, the use of GAMM allowed us to evaluate the trait value (fixed effect) as a smooth non-parametric function of temperature, while accounting for variance that was due to the differences in species' mean trait value across all temperatures (random effect). A significant random effect term indicates differences in the temperature response among individual phytoplankton species. Because species have different temperature optima, we standardized the temperature so that all species had their $R^{*}$ minimum and maximum (for all other traits) at the same position on the temperature axis (set to 0 ). All analyses were performed in the language environment R version 3.2.2 (R Development Core Team, 2015), using gamm4 package.

Second, to measure the temperature sensitivity of each trait, we quantified how steeply its values rise or fall with increasing temperature, by breaking each curve into portions below and above the trait maximum (or minimum for $R^{*}$ ) if the trait showed a non-linear response to temperature. To characterize the rising and falling parts of the curve above or below the trait maximum or minimum for $R^{*}$ (set to 0 on the temperature axis) we fit a linear model with log-transformed trait estimate as the response, which is equivalent to assuming that the trait increases or decreases exponentially with temperature. For the traits that showed a linear response, we fit a linear model to the entire standardized temperature range. We used the estimated slope to calculate a $Q_{10}$ coefficient, representing the temperature sensitivity of the change in the trait value due to an increase in temperature of $10^{\circ} \mathrm{C}$. For the analyses, we only used $I_{\text {opt }}$ estimates when the estimated $I_{o p t}$ was less than the maximum irradiance used in the experiment.

\subsection{Simulation of temperature-dependent competition}

We then used the experimentally derived temperature-dependent competition parameters to compare the outcomes of competition for light and nitrate in multispecies communities across a gradient of environmental temperatures. Competition for light was described using a light-limited chemostat model (Huisman et al. 2002), which we adapted to incorporate the Eiler-Peeters growth-irradiance curve (to our knowledge, for the first time). Competition for nitrogen was described in a separate model of a nutrient-limited chemostat (Monod 1949). Both models were parameterized at each experimental temperature using values from our light (Table A2) and nutrient (Table A3) experiments. We simulated competition over 1,000 model days for a gradient of light and nutrient conditions. 


\section{RESULTS}

\subsection{Temperature-dependence of minimum light and nitrogen requirements}

Minimum light $\left(I^{*}\right)$ and nitrogen $\left(N^{*}\right)$ requirements of the model phytoplankton species were influenced by experimental temperature (Figs. 1a, b). Minimum light requirements $\left(I^{*}\right)$ of all species combined tended to be both lowest and least variable at intermediate experimental temperatures (Fig. 1c), but the overall smoothed trend was not significant (GAMM, F= 2.071, $\mathrm{p}=0.140 ;$ Fig. 1c). There was also an overall positive relationship between $N^{*}$ and temperature across the model phytoplankton species (GAMM, F= 3.8761, p = 0.039; Fig. 1d). These relationships differed among individual phytoplankton species, as the models that included the random effect of species term described the data better than the models without this random effect.

In order to estimate the temperature sensitivity of $I^{*}$ and $N^{*}$, we divided the temperaturedependent curve for all species combined into the increasing and falling portions, and defined the "optimal temperature" as that at which $I^{*}$ and $N^{*}$ were minimized (Figs. 1c, d). The estimated temperature sensitivities $\left(Q_{10}\right)$ across all species for the increasing portions of the curves for $I^{*}$ and $N^{*}$ were 0.70 and 0.45 respectively $(95 \% \mathrm{CI}$ were $[0.61,0.79]$ and $[0.39,0.51]$ respectively; Table A4). The estimated temperature sensitivity $\left(Q_{10}\right)$ for the falling portions of the curves for $I^{*}$ and $N^{*}$ were 2.25 and 1.01 respectively $(95 \%$ CI were $[2.03,2.50]$ and $[0.86,1.16]$ respectively, Table A4). This shows that species' $I^{*}$ and $N^{*}$ s are more sensitive to lower than-optimal temperatures, than higher-than optimal temperatures (Table A4), indicating an asymmetric response of $I^{*}$ and $N^{*}$ around the optimum. Species $I^{*}$ are also more than twice as sensitive to higher than optimal temperatures compare to $N^{*}$, indicating differences in the sensitivity of species $R^{*}$ to different resource types (Table A4).

Despite the overall patterns in minimum resource requirements, there were interspecific differences in $I^{*}$ and $N^{*}$ responses to experimental temperature (Fig. 2, note different y-axes). Whereas $I^{*}$ and $N^{*}$ showed opposite relationships with temperature for some species (e.g., Synechococcus, Cyclotella, and Scenedesmus), both $I^{*}$ and $N^{*}$ responded consistently to temperature for other species (e.g. Kirchneriella). Pediastrum had low requirements for both resource types across the whole temperature gradient (Fig. 2e).

\subsection{Temperature-dependence of other resource competition traits}

'This article is protected by copyright. All rights reserved.' 
Changes in $R^{*}$ with temperature ultimately arise from the temperature-dependence of the traits that determine $R^{*}$. In our study, the maximum specific growth rates $\left(\mu_{\max }\right)$ for light and nitrogen were positively influenced by temperature, ranging from 0.09 to 1.71 day $^{-1}$ (Figs. 3a, A1, Table A2) and from 0.23 to 1.45 day $^{-1}$ (Figs. 3c, A2, Table A3), respectively. The $\mu_{\max }$ for the light limitation experiment was more sensitive to temperature than it was for the nitrogen experiment; $Q_{10}$ for the increasing portions of the curves were 0.45 for light and 0.22 for nitrogen (Figs. 3b, d, Table A4). Across species, growth rates responded more strongly to initial increases in nitrogen than light availability $(\alpha)$, with larger variation across temperatures for nitrogen (Figs. 3e, g). Response curves for $\alpha$ were non-monotonic under both types of resource limitation (Figs. 3f, h). Although the remaining traits were also temperature-dependent (i.e. the $Q_{10}$ values differed from zero; Figs. 3i-n, Table A4), they showed lower sensitivity to temperature and less difference between the response to light and nitrogen in comparison to $I^{*}$ and $N^{*}$ (Table A4).

\subsection{Outcomes of temperature-dependent competition models}

The model simulations demonstrate that observed temperature-dependence of key parameters have the potential to strongly influence resource competition in multispecies plankton communities (Figs. 4, A4). There were changes in dominance between Scenedesmus (at 15 and $25^{\circ} \mathrm{C}$ ) and Pediastrum (at 20 and $30^{\circ} \mathrm{C}$ ) when competing for nitrogen (Fig. 4, bottom row), as these are the species with the lowest $N^{*}$ values at these temperatures (Fig. 1b). In the case of competition for light, simulations of competition at low temperature $\left(15^{\circ} \mathrm{C}\right)$ show Synechococcus as the dominant competitor, whereas Cyclotella dominated at intermediate temperatures $\left(20\right.$ and $\left.25^{\circ} \mathrm{C}\right)$, and Pediastrum at $30^{\circ} \mathrm{C}$ (Fig. 4, top row). The species with the lowest $I^{*}$ in the chemostat model were competitively dominant, and excluded all other species (Fig.

A4, bottom row). Importantly, the relevant $I^{*}$ values in this model differ somewhat from those reported in Fig. 1, because we consider light attenuation across the chemostat. Unlike nutrients, light cannot be uniformly mixed within a water column and each species' production depends jointly on their nonlinear light response curves (eq. 1, Fig. A1) and the light profile across the chemostat (Huisman et al. 2002). One consequence of this difference is that $I^{*}$ can change with the level of irradiance supplied, $\mathrm{I}_{\text {in }}$ (Fig. A4; details on calculating $I^{*}$ also appear in the supplementary material). This subtle effect did not alter the identity of the dominant competitor for light, with the exception of $30^{\circ} \mathrm{C}$, where Pediastrum has the lowest $I^{*}$ below an $\mathrm{I}_{\text {in }}$ of $\sim 55 \mu \mathrm{mol}$ photons $\mathrm{m}^{-2} \mathrm{~s}^{-1}$, while Cyclotella is the better competitor at higher light levels. 


\section{DISCUSSION}

We provide experimental evidence for the temperature-dependence of minimum resource requirements for light $\left(I^{*}\right)$ and nitrogen $\left(N^{*}\right)$. Although minimum resource requirements for light tended to be lowest at intermediate experimental tempertures (Fig. 1c), in agreement with theoretical expectations (e.g., Tilman 2004), there was strong variation in temperature responses across individual species. The minimum resource requirement for nitrogen on average increased at the highest temperatures (Fig. 1d), possibly suggestive of an asymmetric response of $N^{*}$ around its minimum. The model simulations demonstrate that the species-specific differences in the temperature-dependence of $R^{*}$ and other parameters should alter the competitive hierarchies in multispecies communities across a temperature gradient. This is in line with two earlier studies showing that two species of diatoms are superior competitors for silica at different temperatures (Tilman et al. 1981) and that temperature alters the identity of the best rotifer competitor (Stelzer 1998). The partitioning of the temperature-light niche observed in our study may enhance coexistence and biodiversity in environments with temporal or spatial variation in temperature and light (e.g. Descamps-Julien and Gonzalez 2005).

The species-specific responses of $I^{*}$ and $N^{*}$ to temperature indicate distinct interactive effects of temperature and light or nitrogen on each species' population growth rates. Previous tests of combined temperature and nutrient impacts on individual species (Descamps-Julien and Gonzalez 2005, Thomas et al. 2017) showed that temperature and nutrients could limit species ranges by decreasing individual growth rates. Moreover, temperature can also alter the supply ratio of limiting nutrients (Tilman et al. 1986) available in the environment, resulting in the taxonomic replacement and turnover of dominant species (Hillebrand 2011, Kratina et al. 2012). The temperature-dependence of $R^{*}$ and other competitive traits can thus alter community composition, by switching competitive hierarchies under future climate warming.

The monotonic increase in minimum nitrogen requirements with rising temperature indicates that the optimum temperature for $N^{*}$ may not have been captured in the temperature range tested in our study. Maximum growth rate $\left(\mu_{\max }\right)$ also tended to increase monotonically with temperature, whereas $\alpha$ appeared to have a unimodal relationship with temperature for both resources. The non-linear relationship of growth traits with temperature has also been recognised in the minimum silica requirements of two diatom species (Tilman et al. 1981). Furthermore, a synthesis of published light curves showed that $\mu_{\max }$, 
$\alpha$ and $I_{\text {opt }}$ all show unimodal relationships with temperature (Edwards et al. 2016) and a similar pattern of $\mathrm{R}^{*}$ across temperatures is found in models of temperature-nutrient interactions (Follows et al. 2007, Thomas et al. 2017). However, these models presently do not account for the temperature-dependence of traits such as $\alpha$, and may need to be modified accordingly.

Two of our focal species (Kichneriella and Microcystis) were not favored by any of the combinations of light, nitrogen, and temperature we considered. It is possible that these species are favored by conditions outside the range of our study. For example, the $I^{*}$ of Microcystis declined with temperature, suggesting it might become a dominant competitor at temperatures $>30^{\circ} \mathrm{C}$ (Fig. 1a). Alternatively, while Kirchneriella never had the lowest $I^{*}$, it had the highest growth rate of all six species at $20^{\circ} \mathrm{C}$ under $20 \mu \mathrm{mol}$ photons $\mathrm{m}^{-2} \mathrm{~s}^{-1}$ (Fig. A4, middle row). This allowed it to reach high densities and to dominate the community at the beginning of the simulation (Fig. 4). Under fluctuating light conditions, Kirchneriella might be able to persist within a phytoplankton community via a well-documented gleaneropportunist tradeoff (Litchman and Klausmeier 2001, Kremer and Klausmeier 2013) or through other factors, such as predation. It is possible that similar effects might also occur for nitrogen competition, given interspecific differences in maximum growth rates (Fig. A4, top row). Positive growth rates in the absence of light (implying heterotrophic growth) was only observed in Cyclotella at $20^{\circ} \mathrm{C}$ and for Synechococcus at $15^{\circ} \mathrm{C}$; these two taxa were dominant competitors at their respective temperatures. However, these non-zero estimates of growth should be interpreted with caution, as these might have resulted from variation in experimental growth estimates. Interestingly, our model simulations showed that harmful cyanobacterium Microcystis did not dominate phytoplankton assemblages under any experimental temperature, while other cyanobacterium Synechococcus outcompeted other species only at low temperatures. This may suggest weaker cyanobacterial blooms then expected (Paerl and Huisman 2008) at the levels of warming tested in our study or other cyanobacteria species dominating future phytoplankton communities.

This study focuses on the temperature-driven consequences of competitive interactions, which are though to be major structuring force in many ecological communities. However, forecasting multispecies community dynamics in natural ecosystems is challenging due to the complexity of environmental conditions and dynamics at any particular site. Plankton communities undergo strong seasonal successions, where roles of trophic interactions, food quality, the microbial loop and parasites need to be considered (Sommer et al. 1986, 2012). Still, the mechanistic understanding of community 
dynamics in natural ecosystems necessitates understanding competition across environmental gradients as a stepping stone.

Despite the potential for a temperature-dependent resource competition theory to improve forecasting of community dynamics, experimental characterization of resource requirements for a large number of taxa is not practical (Kremer et al. 2017b). However, the integration of nutrient-based competition models with metabolic-based theory (Brown et al. 2004) may be a critical step towards understanding fundamental constraints governing community and ecosystem dynamics under changing climate (Allen and Gillooly 2009). Recent efforts to understand how temperature influences cell physiology and metabolism have shown that nitrogen-rich photosynthetic proteins are less sensitive to temperature changes than phosphorus-rich ribosomes (Toseland et al. 2013, Daines et al. 2014).

Consequently, the activity of ribosomes increases more rapidly with warming than that of photosynthesis proteins, requiring more photosynthetic proteins per cell with warming. This may explain temperatureinduced increases in the nitrogen content of phytoplankton biomass, relative to phosphorus content (Yvon-Durocher et al. 2017). Similarly, the temperature-dependence of four metabolic traits enabled the correct prediction of $72 \%$ of competition experiments between pairs of phytoplankton species (Bestion et al. 2018). Such mechanistic insights may therefore allow the identification of generalities governing the temperature dependencies and sensitivities of species' resource requirement. Efforts to merge metabolic theory with resource competition theory (Ward et al. 2017) can improve a general understanding of the environmental dependence of community dynamics.

Our study demonstrates differential temperature sensitivity of competition for resources across phytoplankton species from varying taxonomic groups. These changes in competitive traits have the potential to reorganize ecological communities across different environmental temperatures that will likely apply to other types of organisms and ecosystems under future climate change. We believe that theoretical and empirical work integrating temperature's influence on physiological processes with resource competition would form a critical step towards understanding and forecasting community and ecosystem dynamics.

Acknowledgements - Funding - PK received funding from the Newton Advanced Fellowships, NAFIR2\180791. CTK was supported by NSF grant OCE-1638958 to Elena Litchman. This is W. K. 
Kellogg Biological Station contribution no. 2136. MKT received funding from the European Union's

Horizon 2020 research and innovation programme under the Marie Skłodowska-Curie grant agreement

TROPHY No. 794264. The Centre for Ocean Life is a VKR Centre of Excellence funded by the Villum

Foundation.

Author contributions - PK, LLP and AN contributed equally to this work. PK and AN conceived the idea, PK, AN and LLP designed the study, LLP and HV collected the data, CK performed the model simulations, LLP, MKT, CK, PK and AN analyzed the data and wrote the paper. All authors gave final approval for publication. 


\section{REFERENCES}

Allen, A. P. and Gillooly, J. F. 2009. Towards an integration of ecological stoichiometry and the metabolic theory of ecology to better understand nutrient cycling. - Ecol. Lett. 12: 369-384.

Allen, A. P. et al. 2005. Linking the global carbon cycle to individual metabolism. - Funct. Ecol. 19: 202213.

Bestion, E. et al. 2018. Metabolic traits predict the effects of warming on phytoplankton competition. Ecol. Lett. 21: 655-664.

Brown, J. H. et al. 2004. Toward a metabolic theory of ecology. - Ecology 85: 1771-1789.

Chase, J. M. and Leibold, M. A. 2003. Ecological niches: linking classical and contemporary approaches. - University of Chicago Press.

Chesson, P. 2000. Mechanisms of maintenance of species diversity. - Annu. Rev. Ecol. Syst. 31: 343366.

Cross, W. F. et al. 2015. Interactions between temperature and nutrients across levels of ecological organization. - Glob. Chang. Biol. 21: 1025-1040.

Daines, S. J. et al. 2014. Multiple environmental controls on phytoplankton growth strategies determine adaptive responses of the N:P ratio. - Ecol. Lett. 17: 414-425.

Dell, A. I. et al. 2011. Systematic variation in the temperature dependence of physiological and ecological traits. - Proc. Natl. Acad. Sci. 108: 10591-10596.

Descamps-Julien, B. and Gonzalez, A. 2005. Stable coexistence in a fluctuating environment: an experimental demonstration. - Ecology 86: 2815-2824.

Dybzinski, R. and Tilman, D. 2007. Resource use patterns predict long-term outcomes of plant competition for nutrients and light. - Am. Nat. 170: 305-318.

Edwards, K. F. et al. 2013. Functional traits explain phytoplankton responses to environmental gradients across lakes of the United States. - Ecology 94: 1626-1635.

Edwards, K. F. et al. 2016. Phytoplankton growth and the interaction of light and temperature: a synthesis at the species and community level. - Limnol. Oceanogr. 61: 1232-1244.

Eilers, P. H. C. and Peeters, J. C. H. 1988. A model for the relationship between light intensity and the rate of photosynthesis in phytoplankton. - Ecol. Model. 42: 199-215.

Eppley, R. W. 1972. Temperature and phytoplankton growth in the sea. - Fish. Bull. 70: 1063-1085.

Field, C. B. et al. 1998. Primary production of the biosphere: integrating terrestrial and oceanic 
components. - Science 281: 237-240.

Follows, M. J. et al. 2007. Emergent biogeography of microbial communities in a model ocean. - Science 315: 1843-1846.

Gillooly, J. F. et al. 2001. Effects of size and temperature on metabolic rate. - Science 293: 2248-2252.

Hillebrand, H. 2011. Temperature mediates competitive exclusion and diversity in benthic microalgae under different N:P stoichiometry. - Ecol. Res. 26: 533-539.

Huisman, J. et al. 2002. Principles of the light-limited chemostat: theory and ecological applications. Antonie Van Leeuwenhoek 81: 117-133.

Keddy, P. 2002. Competition. - Kluwer Academic Publisher.

Kilham, S. S. et al. 1998. COMBO: a defined freshwater culture medium for algae and zooplankton. Hydrobiologia 377: 147-159.

Kingsolver, J. G. 2009. The well-temperatured biologist. - Am. Nat. 174: 755-768.

Kratina, P. et al. 2012. Warning modifies trophic cascades and eutrophication in experimental freswater communities. - Ecology 93: 1421-1430.

Kremer, C. T. and Klausmeier, C. A. 2013. Coexistence in a variable environment: eco-evolutionary perspectives. - J. Theor. Biol. 339: 14-25.

Kremer, C. T. et al. 2017a. Temperature- and size-scaling of phytoplankton population growth rates: reconciling the Eppley curve and the metabolic theory of ecology. - Limnol. Oceanogr. 62: 16581670.

Kremer, C. T. et al. 2017b. Realizing the potential of trait-based aquatic ecology: new tools and collaborative approaches. - Limnol. Oceanogr. 62: 253-271.

Lehman, C. and Tilman, D. 2000. Biodiversity, stability, and productivity in competitive communities. Am. Nat. 156: 534-552.

Litchman, E. and Klausmeier, C. A. 2001. Competition of phytoplankton under fluctuating light. - Am. Nat. 157: 170-187.

Litchman, E. and Klausmeier, C. A. 2008. Trait-based community ecology of phytoplankton. Annu. Rev. Ecol. Syst., 39: 615-639.

Macarthur, R. and Levins, R. 1967. The limiting similarity, convergence, and divergence of coexisting species. - Am. Nat. 101: 377-385.

Martiny, A. C. et al. 2013. Strong latitudinal patterns in the elemental ratios of marine plankton and 
organic matter. - Nat. Geosci. 6: 279.

Miller, T. E. et al. 2005. A critical review of twenty years' sse of the Resource- Ratio Theory. - Am. Nat. 165: 439-448.

Monod, J. 1949. The growth of bacterial cultures. - Annu. Rev. Microbiol. 3: 371-394.

Narwani, A. et al. 2015. Common ancestry is a poor predictor of competitive traits in freshwater green algae. - PLoS One 10: 1-18.

O’Connor, M. I. 2009. Warming strengthens an herbivore-plant interaction. - Ecology 90: 388-398.

Paerl, H. W. and Huisman, J. 2008. Blooms like it hot. - Science 320: 57-58.

Savage, V. M. et al. 2004. Effects of body size and temperature on population growth. - Am. Nat. 163: $429-441$.

Schaum, C.-E. et al. 2017. Adaptation of phytoplankton to a decade of experimental warming linked to increased photosynthesis. - Nat. Ecol. \&Amp; Evol. 1: 94.

Sentis, A. et al. 2017. Temperature-size responses alter food chain persistence across environmental gradients. - Ecol. Lett. 20: 852-862.

Shatwell, T. et al. 2014. Temperature and photoperiod interactions with phosphorus-limited growth and competition of two diatoms. - PLoS One 9: 1-15.

Shurin, J. B. et al. 2012. Warming shifts top-down and bottom-up control of pond food web structure and function. - Philos. Trans. R. Soc. London B Biol. Sci. 367: 3008-3017.

Sommer, U., Gliwicz, Z. M., Lampert, W. and Duncan, A. 1986. The PEG-model of seasonal succession of planktonic events in fresh waters. Arch. fur Hydrobiol., 106: 433-471.

Sommer, U., Adrian, R., De Senerpont Domis, L., Elser, J. J., Gaedke, U., Ibelings, B., Jeppesen, E., Lürling, M., Molinero, J. C., Mooij, W. M., van Donk, E. and Winder, M. 2012. Beyond the plankton ecology group (PEG) model: mechanisms driving plankton succession. Annu. Rev.

Ecol. Syst., 43: 429-448.

Stelzer, C.-P. 1998. Population growth in planktonic rotifers. Does temperature shift the competitive advantage for different species? - Hydrobiologia 387388: 349-353.

Sterner, R. W. and Elser, J. J. 2002. Ecological stoichiometry: the biology of elements from molecules to the biosphere. - Princeton University Press.

Thomas, M. K. et al. 2016. Environment and evolutionary history determine the global biogeography of phytoplankton temperature traits. - Glob. Ecol. Biogeogr. 25: 75-86.

'This article is protected by copyright. All rights reserved.' 
Thomas, M. K. et al. 2017. Temperature-nutrient interactions exacerbate sensitivity to warming in phytoplankton. - Glob. Chang. Biol. 23: 3269-3280.

Tilman, D. 1977. Resource competition between plankton algae: an experimental and theoretical approach. - Ecology 58: 338-348.

Tilman, D. 1980. Resources: A graphical-mechanistic approach to competition and predation. - Am. Nat. 116: $362-393$.

Tilman, D. 1981. Tests of resource competition theory using four species of Lake Michigan algae. Ecology 62: 802-815.

Tilman, D. 1982. Resource competition and community structure. - Princeton University Press.

Tilman, D. 2004. Niche tradeoffs, neutrality, and community structure: a stochastic theory of resource competition, invasion, and community assembly. - Proc. Natl. Acad. Sci. 101: 10854-10861.

Tilman, D. et al. 1981. Competition and nutrient kinetics along a temperature gradient: an experimental test of a mechanistic approach to niche theory. - Limnol. Oceanogr. 26: 1020-1033.

Tilman, D. et al. 1986. Green, bluegreen and diatom algae: taxonomic differences in competitive ability for phosphorus, silicon and nitrogen. - Arch. fur Hydrobiol. 106: 473-485.

Toseland, A. et al. 2013. The impact of temperature on marine phytoplankton resource allocation and metabolism. - Nat. Clim. Chang. 3: 979-984.

van Donk, E. and Kilham, S. S. 1990. Temperature effects on silicon- and phosphorus-limited growth and competitive interactions among three diatoms. - J. Phycol. 26: 40-50.

Ward, B. A. et al. 2017. The size dependence of phytoplankton growth rates: a trade-off between nutrient uptake and metabolism. - Am. Nat. 189: 170-177.

Wilson, J. B. et al. 2007. Is there really insufficient support for Tilman's R* concept? A Comment on Miller et al. - Am. Nat. 169: 700-706.

Yuan, Z. Y. and Chen, H. Y. H. 2015. Decoupling of nitrogen and phosphorus in terrestrial plants associated with global changes. - Nat. Clim. Chang. 5: 465.

Yvon-Durocher, G. et al. 2010. Warming alters the metabolic balance of ecosystems. - Philos. Trans. R. Soc. B Biol. Sci. 365: 2117-2126.

Yvon-Durocher, G. et al. 2015. Temperature and the biogeography of algal stoichiometry. - Glob. Ecol. Biogeogr. 24: 562-570.

Yvon-Durocher, G. et al. 2017. The temperature dependence of phytoplankton stoichiometry: 
investigating the roles of species sorting and local adaptation. - Front. Microbiol. 8: 2003.

'This article is protected by copyright. All rights reserved.' 


\section{FIGURE LEGENDS}

Figure 1. Temperature alters the minimum requirements for light $\left(I^{*}\right)$ and nitrogen $\left(N^{*}\right)$ of six common phytoplankton species. Individual phytoplankton species are denoted by different symbols and colours. Panels a and $\mathbf{b}$ show within-species patterns in $I^{*}$ and $N^{*}$ across temperature. Panels $\mathbf{c}$ and $\mathbf{d}$ show across-species patterns in $I^{*}$ and $N^{*}$ across temperature, using GAMMs. The plots of across-species patterns (c \& d) differ in: (1) standardizing the x-axis so that all species' minimum trait values align at the same value $\left(0^{\circ} \mathrm{C}\right)$, and (2) accounting for interspecific differences in mean trait value across a temperature gradient using a random effect. Significant (non-significant at $\alpha=0.05$ ) smoothed trends are indicated by solid (dashed) lines, and shaded bands show $\pm 1 \mathrm{SE}$.

\section{Within-species patterns}

(a)

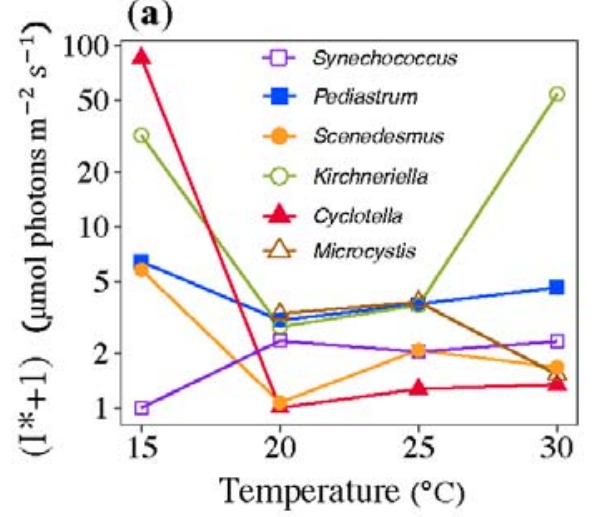

(b)

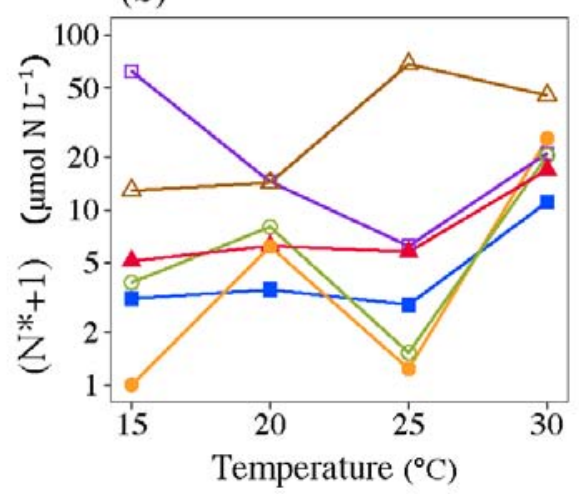

\section{Across-species patterns}

(c)

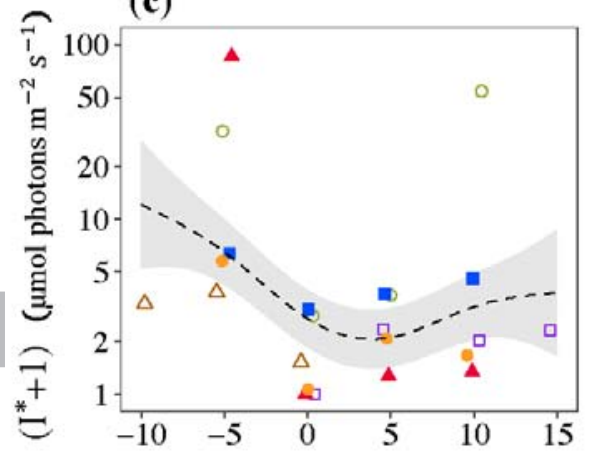

Temperature relative to $\min \left(\mathrm{I}^{*}\right)\left({ }^{\circ} \mathrm{C}\right)$ (d)

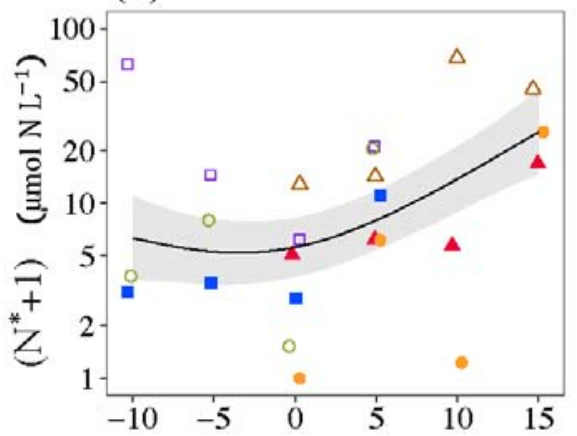

Temperature relative to $\min \left(\mathrm{N}^{*}\right)\left({ }^{\circ} \mathrm{C}\right)$

'This article is protected by copyright. All rights reserved.' 
Figure 2. Temperature alters within-species requirements for light $\left(I^{*}\right)$ and nitrogen $\left(N^{*}\right) . I^{*}$ and $N^{*}$ show opposite relationship with temperature for some species (a, c, f), or similar trends for other species (d). Note different y-axes across the panels.
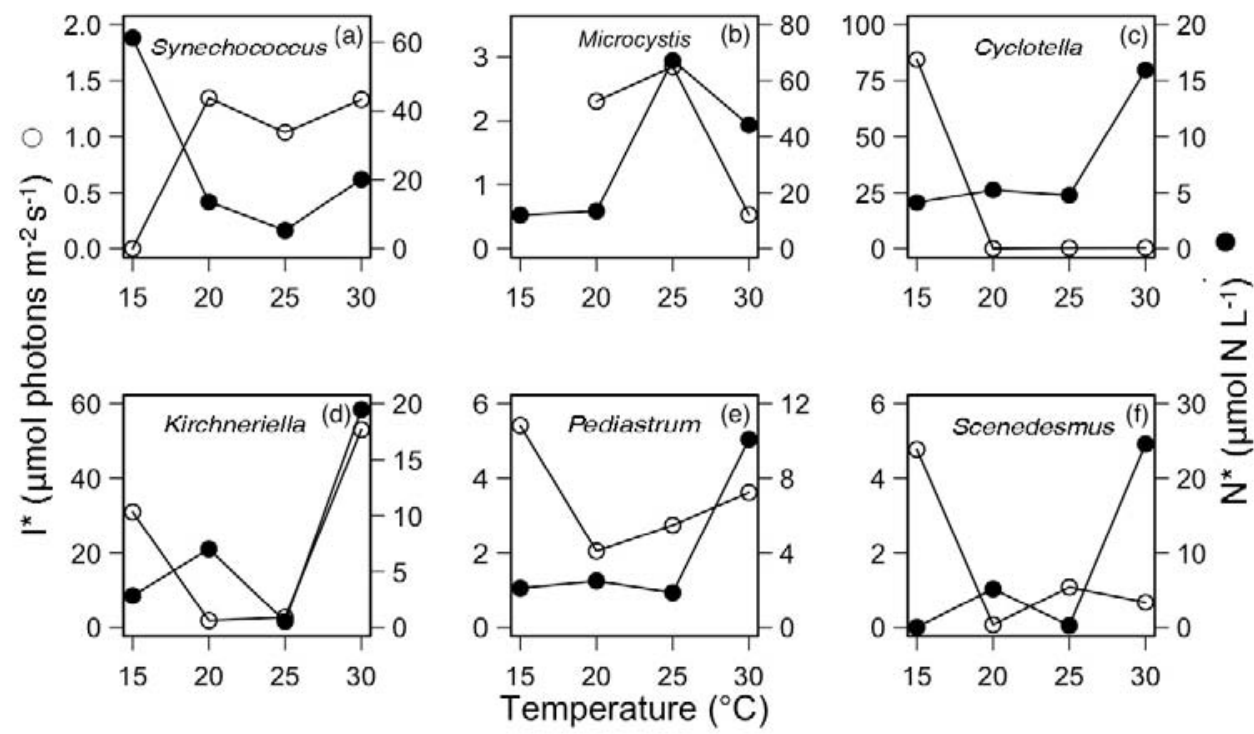

'This article is protected by copyright. All rights reserved.' 
Figure 3. The key traits for nitrogen and light competition depend on experimental temperature. The effect of temperature on maximum growth rate under optimal light levels (i.e., $I=I_{\text {opt }}$, and without adjusting for heterotrophic growth, if any) $(\mathbf{a}, \mathbf{b})$ and unlimited nitrate $(\mathbf{c}, \mathbf{d})$. The effect of temperature on the initial slope of the light growth curve, $\alpha(\mathbf{e}, \mathbf{f})$ and of the nitrogen growth curve $(\mathbf{g}, \mathbf{h})$. The effect of temperature on the specific growth rate at $I=0$, i.e. in the absence of light (parameter $h$ ), implying heterotrophic growth $(\mathbf{i}, \mathbf{j})$, on the species background mortality, $m(\mathbf{k}, \mathbf{l})$ and on the optimal irradiance for growth, $I_{\text {opt }}(\mathbf{m}, \mathbf{n})$. Panels $\mathbf{a}, \mathbf{c}, \mathbf{e}, \mathbf{g}, \mathbf{i}, \mathbf{k}, \mathbf{m}$ show within-species patterns in irradiance and nitrogen competition traits across temperature. Panels $\mathbf{b}, \mathbf{d}, \mathbf{f}, \mathbf{h}, \mathbf{j}, \mathbf{l}, \mathbf{n}$ show across-species patterns in irradiance and nitrogen competition traits across temperature, using GAMMs. Significant (non-significant at $\alpha=$ 0.05) smoothed trends are indicated by solid (dashed) lines, and shaded bands show $\pm 1 \mathrm{SE}$. The plotted data points are corrected to remove differences between species in the mean trait value across temperatures. The $\mathrm{x}$-axis represents temperature values that have been standardized so that all species had their trait maximum at the same position $\left(0{ }^{\circ} \mathrm{C}\right)$.
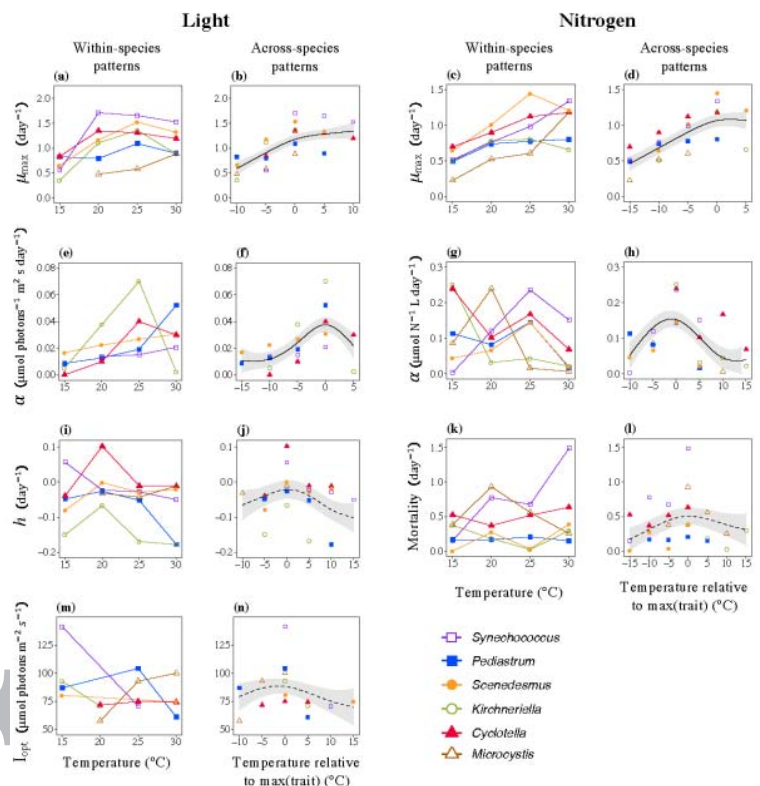

- cydotente

$\triangle$ Microystio

'This article is protected by copyright. All rights reserved.' 
Figure 4. Temperature alters competitive outcomes in multispecies phytoplankton assemblages.

Simulated population dynamics reveal which species dominate during initial transient dynamics (fast growing species) as well as over the long-term (superior competitors) given competition for light (top row) and nitrogen (bottom row) at each experimental temperature. Results are based on chemostat models (eqs. A3 and A4) parameterized using the population growth experiments (Tables A2, A3) and run for 1000 model days. In all cases, a single species dominates by the end of the simulations, while all others are driven to low densities and excluded. The identity of the dominant species changes between temperatures. Comparisons between $R^{*}$ values and growth rates for each species at each temperature in these simulations appear in the Supplementary material (Appendix 1, Fig. A4).

(a) $15^{\circ} \mathrm{C}$

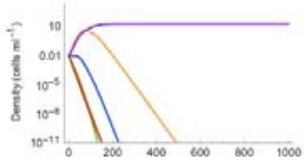

(e) $15^{\circ} \mathrm{C}$

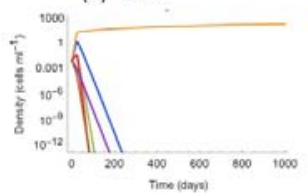

(b) $20^{\circ} \mathrm{C}$

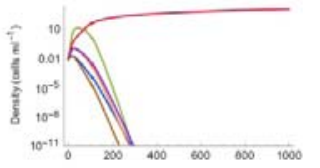

Nitrogen

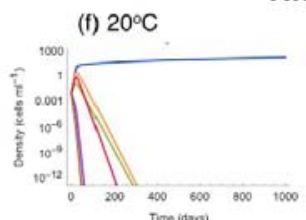

Cyclotella

- Scenedesmus - Synechococcus

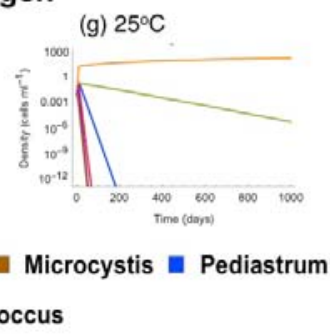

(c) $25^{\circ} \mathrm{C}$

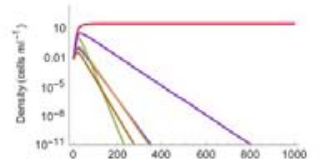

(g) $25^{\circ} \mathrm{C}$ (d) $30^{\circ} \mathrm{C}$

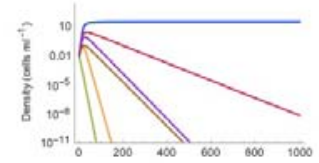

(h) $30^{\circ} \mathrm{C}$

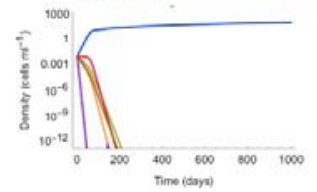

'This article is protected by copyright. All rights reserved.' 